\title{
Cutaneous Carcinomatous Arteriopathy and Retiform Purpura Secondary to Metastatic Penile Carcinoma
}

\author{
Zachary A. Carter, MD; Gerardo Marrazzo, MD; Blake Galler, DO; Arturo R. Dominguez, MD
}

\section{PRACTICE POINTS}

- Cutaneous metastases may present in multiple ways, including carcinoma erysipeloides, carcinoma en cuirasse, or carcinoma telangiectoides.

- Ischemic cutaneous lesions, characterized by livedoid skin changes and retiform purpura, occur less commonly in the setting of malignancy.

- Direct mechanisms include carcinomatous arteriopathy and vasculitis carcinomatosa. Indirect systemic processes include coagulopathies such as disseminated intravascular coagulation, thrombotic thrombocytopenia purpura, catastrophic antiphospholipid antibody syndrome, calciphylaxis, and cryoglobulinemia.

\section{To the Editor:}

A 56-year-old man with a history of stage IV metastatic penile squamous cell carcinoma treated with penectomy and chemotherapy with 5-fluorouracil and cisplatin presented with several painful ulcerations in the groin, abdomen, and thighs. The lesions initially appeared in the groin and were treated as bacterial abscesses with antibiotics. Over the next few weeks, new lesions appeared on the abdomen and thighs. An additional cycle of chemotherapy led to a reduction in number; however, they again increased within a few weeks. Medications included enoxaparin followed by 3 weeks of warfarin use due to a right leg deep vein thrombosis.

Physical examination revealed multiple 1 - to $4-\mathrm{cm}$, firm, ulcerated nodules on the bilateral inguinal folds, abdomen, and upper thighs, as well as areas of livedo racemosa and noninflammatory retiform purpura with central ulceration (Figures 1 and 2). This retiform purpura was both perilesional and in areas without ulcerations. Laboratory values included the following: sodium, $127 \mathrm{mmol} / \mathrm{L}$ (reference range, 136-145 mmol/L); prothrombin time, 16.1 seconds (reference range, 11-15 seconds); white blood cell count, $20.69 \times 10^{9} / \mathrm{L}$ (reference range, $4.5-11.0 \times 10^{9} / \mathrm{L}$ ) with $87 \%$ neutrophils (reference range, 54\%-62\%); hemoglobin, $6.1 \mathrm{~g} / \mathrm{dL}$ (reference range, $13.5-17.5 \mathrm{~g} / \mathrm{dL}$ ); hematocrit, $18.8 \%$ (reference range, $41 \%-53 \%$ ); platelets, $474 \times 10^{9} / \mathrm{L}$ (reference range, $150-400 \times 10^{9} / \mathrm{L}$ ); D-dimer, $0.77 \mathrm{mg} / \mathrm{L}$ (reference range, $\leq 0.50 \mathrm{mg} / \mathrm{L}$ ); fibrinogen, $489 \mathrm{mg} / \mathrm{dL}$ (reference range, 150-400 $\mathrm{mg} / \mathrm{dL}$ ); prior urine culture positive for Pseudomonas aeruginosa. He was negative for hepatitis B and hepatitis C viruses as well as HIV, and the lesions were not clinically consistent with herpes simplex virus, as they were not scalloped or circinate. Punch biopsies were obtained from a nodule on the left leg and a purpuric patch on the right leg.

Dr. Carter is from the University of Cincinnati Medical Center, Ohio. Dr. Marrazzo is from the Skin Surgery Center, Hickory, North Carolina.

Dr. Galler is from the Alaska Veterans Affairs Healthcare System, Anchorage. Dr. Dominguez is from the University of Texas Southwestern Medical Center, Dallas.

The authors report no conflict of interest

Correspondence: Arturo R. Dominguez, MD, University of Texas Southwestern Medical Center, Departments of Dermatology and Internal Medicine, 5323 Harry Hines Blvd, Dallas, TX 75390-9069 (Arturo.Dominguez@UTSouthwestern.edu).

doi: $10.12788 /$ cutis.0304 


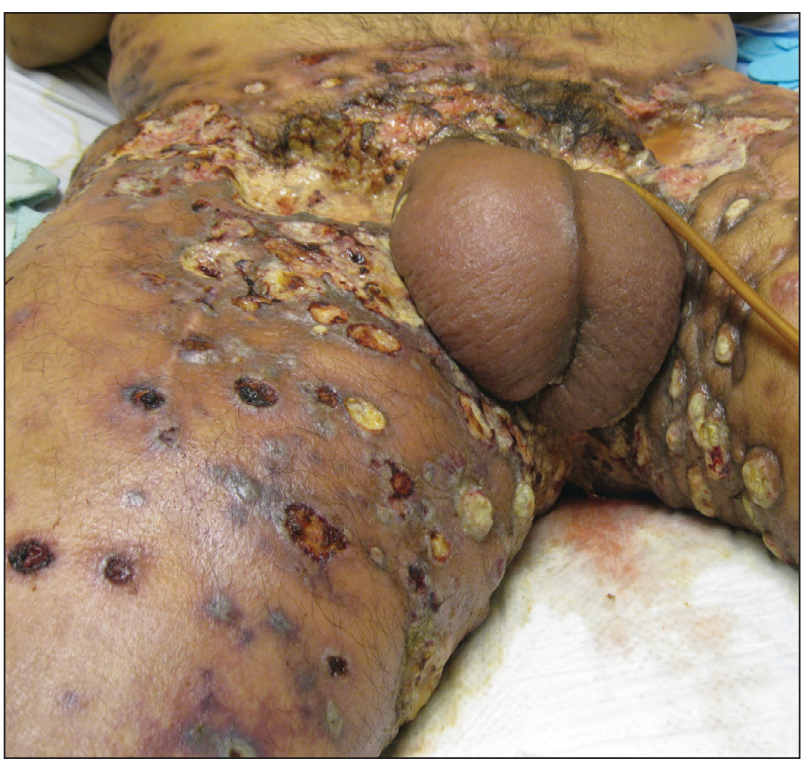

FIGURE 1. Ulcerated nodules and retiform purpura with ulceration on the upper legs, groin, and abdomen following a penectomy.

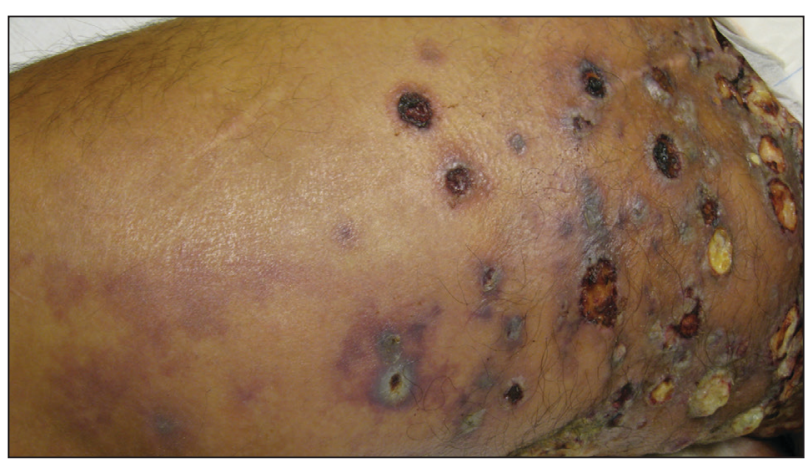

FIGURE 2. Livedo racemosa on the inner right leg without accompanying ulceration.

Histopathology of the ulcerated nodule revealed a proliferation of atypical keratinocytes with hyperchromatic and pleomorphic nuclei in the dermis without involvement of the overlying epidermis, consistent with metastatic squamous cell carcinoma (Figure 3). Histopathology of the purpuric patch demonstrated a thrombotic vasculopathy with numerous fibrin thrombi in the lumina of superficial dermal capillaries (Figure 4). No atypical cells, calcifications, or organisms were seen in the vessels. Periodic acid-Schiff, Fite, and Gram stains also were negative. The extent of the disease portended a poor prognosis, and additional vasculopathic workup was not pursued. Following antibiotic treatment and palliative care consultation, he died from subsequent infectious complications 1 month after presentation.

Cutaneous metastases may occur in the setting of multiple malignancies including breast, lung, melanoma, and various gastrointestinal cancers. ${ }^{1}$ These may present

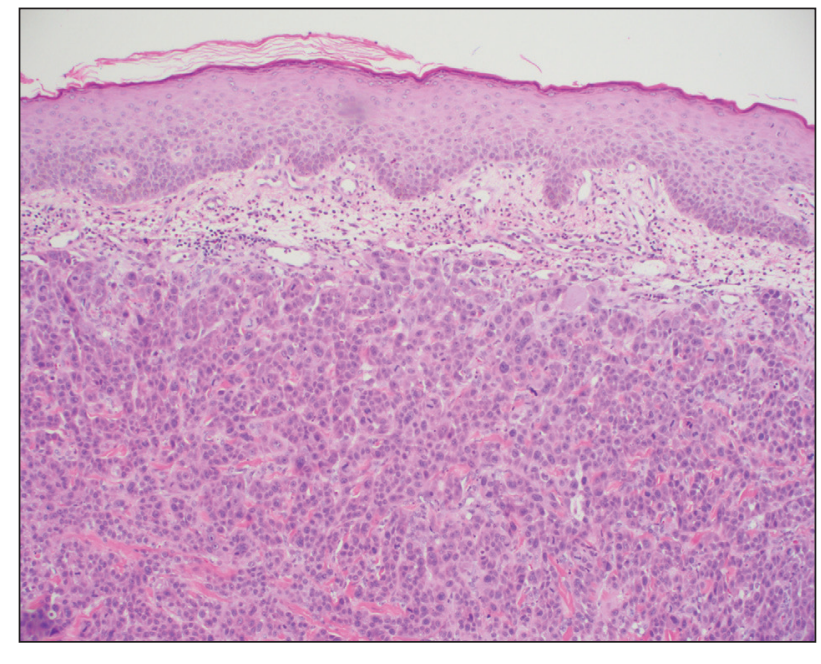

FIGURE 3. Punch biopsy of a nodule on the left thigh revealed a proliferation of atypical keratinocytes seen throughout the dermis without an epidermal connection, representing metastatic squamous cell carcinoma (H\&E, original magnification $\times 100)$.

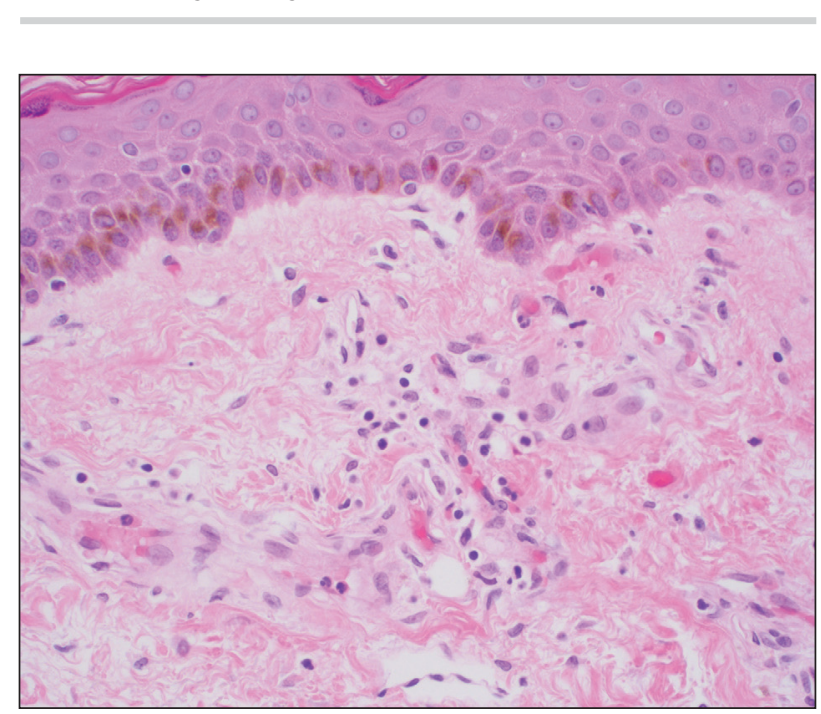

FIGURE 4. Punch biopsy of purpura on the right thigh revealed fibrin thrombi in multiple small blood vessels throughout the dermis with no evidence of inflammation, representing thrombotic vasculopathy $(H \& E$, original magnification $\times 200)$.

in multiple ways, including firm nontender nodules or as plaques with one of the following morphologies: carcinoma erysipeloides: erythematous, occasionally tender areas resembling cellulitis due to lymphatic obstruction by tumor cells; ; carcinoma en cuirasse: indurated sclerotic scarlike plaques due to collagen infiltration ${ }^{3}$; or carcinoma telangiectoides: telangiectatic, thin erythematous plaques due to dermal capillary infiltration by malignant cells. ${ }^{3}$

Ischemic cutaneous lesions less commonly occur in the setting of malignancy and can be the result of both direct and indirect systemic effects from the cancer. Malignancies are known to directly trigger vasculopathies in other organs, most commonly the lungs, through 
2 primary mechanisms. First, in carcinomatous arteriopathy, metastatic cells promote fibrocellular intimal proliferation of small pulmonary arteries and arterioles leading to stenosis, thrombosis, and obliteration. This mechanism has been described in pulmonary thrombotic microangiopathy secondary to lung carcinoma. ${ }^{4}$ This pathophysiology likely is also what underlies paraneoplastic acral vascular syndromes, which culminate in digital ischemia. Hypothesized mechanisms for this ischemia also range from vasospasm to thromboembolism. ${ }^{5}$ Secondly, in vasculitis carcinomatosa, metastatic tumor cells damage or block vessel walls, resulting in end-organ ischemia. Vasculitis carcinomatosa is a well-known phenomenon in angiocentric and intravascular lymphoid malignancies (typically of B-T or natural killer/T-cell origin) but also has been reported in a case of gastric adenocarcinoma with arterial invasion. ${ }^{6}$ This process is different than carcinoma telangiectoides where malignant cells may be present in the vasculature on histopathology but not trigger thrombosis and ischemic skin necrosis.

Systemic coagulopathies such as disseminated intravascular coagulation (DIC), thrombotic thrombocytopenia purpura, and catastrophic antiphospholipid antibody syndrome can occur in the setting of malignancies. ${ }^{7}$ Clinically, all may present with livedo racemosa, noninflammatory retiform purpura, and widespread skin necrosis. In adult patients, purpura fulminans most often is seen in the setting of sepsis and DIC, with accompanying evidence of microangiopathy. ${ }^{8}$ Catastrophic antiphospholipid antibody syndrome can be triggered by malignancy and is characterized by central nervous system, renal, pulmonary, and gastrointestinal complications. Skin involvement such as ulcers, livedo reticularis, and gangrene have been reported. ${ }^{9}$ Other causes of thrombotic vasculopathy include warfarin necrosis, heparin-induced thrombotic thrombocytopenia, calciphylaxis, and angioinvasive infections. ${ }^{8}$ Warfarin necrosis and heparin-induced thrombotic thrombocytopenia typically present days after initiating therapy with the respective medication. Calciphylaxis typically occurs in patients on dialysis, though it may occur in nonuremic patients including those with malignancy. ${ }^{8}$ Patients with malignancies on chemotherapy can become neutropenic and are at risk for ecthyma gangrenosum due to $P$ aeruginosa and other gram-negative rods, Staphylococcus aureus, and angioinvasive fungi. ${ }^{10}$

Based on clinical, histopathological, and laboratory data, we favored a diagnosis of cutaneous carcinomatous arteriopathy. Vasculitis carcinomatosa was a possibility despite the lack of vasculotropism on histopathology, which may have been due to biopsy site selection.
Systemic coagulopathies such as DIC, thrombotic thrombocytopenia purpura, and catastrophic antiphospholipid antibody syndrome were unlikely, as the ischemic skin lesions and livedo racemosa were limited to areas adjacent to cutaneous metastases, and the patient lacked other common multiorgan manifestations or laboratory findings. Although our patient was on warfarin, he was on a stable dose for weeks and histopathologic features of subcutaneous thrombosis were not seen. The biopsy also was not consistent with calciphylaxis. Ecthyma gangrenosum was unlikely given the lack of organisms on histopathology and negative skin and blood cultures. Although additional laboratory testing in this patient may have included cryoglobulins and cryofibrinogens, both entities were unlikely due to a lack of ischemic acral lesions.

In conclusion, we present a case of localized thrombotic vasculopathy that likely was secondary to cutaneous carcinomatous arteriopathy in the setting of cutaneous metastatic penile squamous cell carcinoma. The differential diagnosis of retiform purpura, livedo racemosa, and other signs of cutaneous ischemia in patients with metastatic cancer is broad and can be the result of both direct and indirect systemic effects from the cancer. Appropriate workup in these cases should include skin biopsies for histopathology and culture, medication review, and laboratory evaluation for systemic coagulopathies.

\section{REFERENCES}

1. Alcaraz I, Cerroni L, Ruetten A, et al. Cutaneous metastases from internal malignancies: a clinicopathologic and immunohistochemical review. Am J Dermatopathol. 2012;34:347-393.

2. Prat L, Chouaid C, Kettaneh A, et al. Cutaneous lymphangitis carcinomatosa in a patient with lung adenocarcinoma: case report and literature review. Lung Cancer. 2013;79:91-93.

3. Marneros AG, Blanco F, Husain S, et al. Classification of cutaneous intravascular breast cancer metastases based on immunolabeling for blood and lymph vessels. J Am Acad Dermatol. 2009;60:633-638.

4. von Herbay A, Illes A, Waldherr R, et al. Pulmonary tumor thrombotic microangiopathy with pulmonary hypertension. Cancer. 1990;66:587-592.

5. Besnerais ML, Miranda S, Cailleux N, et al. Digital ischemia associated with cancer. Medicine. 2014;93:E47.

6. Sweeney S, Utzschneider R, Fraire AE. Vasculitis carcinomatosa occurring in association with adenocarcinoma of the stomach. Ann Diagn Pathol. 1998;2:247-249.

7. Zwicker JI, Furie BC, Furie B. Cancer-associated thrombosis. Crit Rev Oncol Hematol. 2007;62:126-136.

8. Thornsberry LA, LoSicco KI, English JC. The skin and hypercoagulable states. J Am Acad Dermatol. 2013;69:450-462.

9. Miesbach W, Asherson RA, Cervera R, et al; CAPS Registry Group. The role of malignancies in patients with catastrophic anti-phospholipid (Asherson's) syndrome. Clin Rheumatol. 2007;26:2109-2114.

10. Pozo D. Ecthyma gangrenosum-like eruption associated with Morganella morganii infection. Br J Dermatol. 1998;139:520-521. 